EPJ Web of Conferences 20, 02006 (2012)

DOI: $10.1051 /$ epjconf/20122002006

(C) Owned by the authors, published by EDP Sciences, 2012

\title{
Photoproduction of charged pions from the deuteron
}

\author{
Hiroki Kanda ${ }^{1, a}$ for the NKS/NKS2 Collaboration \\ Department of Physics, Tohoku University
}

\begin{abstract}
The photoproduction of the charged pions from the deuteron has been studied with the Neutral Kaon Spectrometer (NKS) and the Neutral Kaon Spectrometer 2 (NKS2) at the Research Center for Electron Photon Science, Tohoku University (ELPH) which was previously known as the Laboratory of Nuclear Science (LNS-Tohoku) using the GeV-region tagged photon beam. The total cross section for the quasi-free (QF) $\pi^{+} \pi^{-}$ photoproduction from the deuteron was measured with NKS in the energy region from 0.8 to $1.1 \mathrm{GeV}$. The cross section for the double $\Delta$ excitation in the intermediate state was derived in the analysis of the non-quasi-free (NQF) process, in which two nucleons of the deuteron share the energy of the incident photon. The prospect of the measurements of double pion photoproduction with NKS2 and upgraded NKS2 is discussed.
\end{abstract}

\section{Introduction}

The pion photoproduction from the nucleon has been investigated both experimentally and theoretically for the study of the nucleon resonances for tens of years. The contribution of the double pion photoproduction from the proton is dominant in the energy region higher than $0.6 \mathrm{GeV}$. The $\pi^{+} \pi^{-}$ photoproduction cross section accounts for approximately 30 percent of the total photoproduction in this energy region.

The deuteron is used as the practical neutron target for the processes above the delta resonance region. The reason is the dominance of the quasi-free $(\mathrm{QF})$ process, i.e. only a single nucleon of the deuteron participates in the reaction. However, the contribution of the non-quasi-free (NQF) process in which two nucleons participate in the reaction has to be taken into account in some reaction channels. For the case of the double pion photoproduction, the effect of the final state interaction and inclusive theoretical treatment of the double pion photoproduction on the deuteron is reported in Ref. [1]. In the NQF processes, the significantly large cross section of the double $\Delta$ excitation in the intermediate state is reported in Refs. [2,3]. The double $\Delta$ excitation plays a key role in the intermediate state of the isoscalar two pion production which is known as the ABC effect[4,5]. It was originally observed as the enhancement of the cross section in the invariant spectrum of two pions in the double-pionic fusion of the proton and the deuteron into ${ }^{3} \mathrm{He}$. The further investigation revealed the attractive nature of the $\Delta-\Delta$ interaction [6]. The recent experimental studies of this effect have been carried out by the use of the proton, neutron and deuteron beam and interesting results have been reported[7-9]. The photo-excitation of the double $\Delta$ and the coherent double pion photoproduction on the deuteron will shed another light on the mechanism of this effect.

We have measured the $\pi^{+} \pi^{-}$photoproduction on the deuteron with the NKS and NKS2 at LNSTohoku. The photon energy range was from 0.8 to $1.1 \mathrm{GeV}$. We have measured the cross sections for the QF $\pi^{+} \pi^{-}$photoproduction cross section on the deuteron and the $\Delta^{++} \Delta^{-}$photoproduction. These results with the NKS was reported in Ref. [10]. The analysis of the data taken with NKS2 is in progress.

\footnotetext{
a e-mail: kanda@m.tohoku.ac.jp
} permits unrestricted use, distribution, and reproduction in any noncommercial medium, provided the original work is properly cited. 


\section{EPJ Web of Conferences}

\section{Experiment and Prospect}

The NKS is the magnetic spectrometer constructed at LNS-Tohoku on the tagged photon beam line. The tagged photon beam was provided by the STB-Tagger [11] located in the bending magnet of the Stretcher Booster Synchrotron (STB). The tagged photon energy range was from 0.8 to $1.1 \mathrm{GeV}$ at the electron energy of $1.2 \mathrm{GeV}$. The NKS consisted of a dipole magnet with the maximum flux density of $0.5 \mathrm{~T}$, two sets of drift chambers, and two sets of plastic hodoscopes. We set the cryogenic target system at the center of the pole gap of the dipole magnet. We used the liquid hydrogen and liquid deuterium as the target. The detail of the spectrometer is described elsewhere [12].

We took the data with trigger on more than two charged particles associated with the tagger hit. In the analysis, we required the detection of $\pi^{+}, \pi^{-}$, and proton. For the kinematic selection for the hydrogen target data, the proton missing mass was required to $\gamma X \rightarrow \pi^{+} \pi^{-} p$ reaction. For the deuterium target data, the neutron missing mass was required to $\gamma d \rightarrow \pi^{+} \pi^{-} p X$ reaction. The momentum of the neutron was used for the discrimination of QF and NQF process in the $\gamma d \rightarrow \pi^{+} \pi^{-} p n$ events. The events with the neutron momentum smaller than $0.2 \mathrm{GeV} / \mathrm{c}$ was categorized as QF process and the events with the neutron momentum higher than $0.3 \mathrm{GeV} / \mathrm{c}$ was categorized as NQF process. The detection efficiency were estimated with use of the Monte Carlo simulation with generator of expected subprocesses with the same final state as $\pi^{+} \pi^{-} p$. The invariant mass distributions of the data were fitted with the sum of the simulated invariant masses of all the subprocesses for the derivation of the fraction of each subprocess. Total cross cross section was obtained as the sum of the cross sections for all the subprocesses.

The obtained cross sections for the $\pi^{+} \pi^{-}$photoproduction on the proton with the hydrogen target and the deuterium target are compared to the previous data with the hydrogen target $[13,14]($ Fig. 1). The cross sections for the hydrogen target is consistent with the previous data, However, the QF cross section for the deuterium target is systematically smaller than the previous data. We suspect that the possible reason for this discrepancy is the final state interaction between the pion and the spectator nucleon. In our analysis, events with the neutron with higher momentum than $0.3 \mathrm{GeV} / \mathrm{c}$ after the FSIs are counted as the NQF process. It might reduce the QF cross section. The $\Delta^{++} \Delta^{-}$cross section is compared to the previous data(Fig. 2). The obtained cross section is smaller than the previous data and the energy dependence is quite different. It is important to cover the energy region above the double $\Delta$ photoproduction threshold of $680 \mathrm{MeV}$.

The NKS was replaced by the NKS2 which consisted of the larger spectrometer magnet than the NKS. New sets of the drift chambers and plastic hodoscopes are incorporated in the NKS2. The geometrical acceptance is approximately the same, but NKS2 covers the forward region. The larger pole size of the spectrometer magnet enabled us of the longer tracking length which improved the momentum resolution. It also enabled us of the longer TOF length which improved the particle identification. The momentum resolutions for $0.5 \mathrm{GeV} / \mathrm{c}$ were approximately $5 \%$ and $2 \%$ for NKS and NKS2, respectively. The TOF resolutions were $0.5 \mathrm{~ns}$ and $0.3 \mathrm{~ns}$ for NKS and NKS2, respectively. The detection of the deuteron was enabled by the forward coverage and better resolutions. Fig. 3 shows the spectrum of the $\pi^{+} \pi^{-}$invariant mass by gating on the deuteron, $\pi^{+}$and $\pi^{-}$. The peak at the $\rho$ meson is significant but background distribution can be seen, either. To detect the invariant mass enhancement as the ABC effect, comparison with the simulation for the three body phase space is in progress.

The further upgrade of the NKS2 have completed to increase the geometrical acceptance. Before the upgrade, a measurement of a mass and momentum of a charged particle was possible only for the particle which travels through all the detectors. Thus, it was impossible to detect and identify a particle with high momenta parallel to the magnetic flux before the upgrade. This requirement limited then acceptance. In the upgrade, the inner tracking chamber which consists of eight stereo layers was developed. The inner hodoscope was renewed with double-reading counters to obtain the hit position along the plastic scintillator bar and to increase the timing resolution. They enabled to detect the three dimensional information of the track of the particle which only travels through the inner tracking chamber and hodoscope and goes out of the detector setup. It is expected that the detection efficiency for a charged pion which distribute in the wide angular range is highly improved.

The experiment to measure the cross section in the lower photon energy is planned to take data in two steps of the energy regions. The energy regions are shown as three bars in Figs. 1 and 2. The 


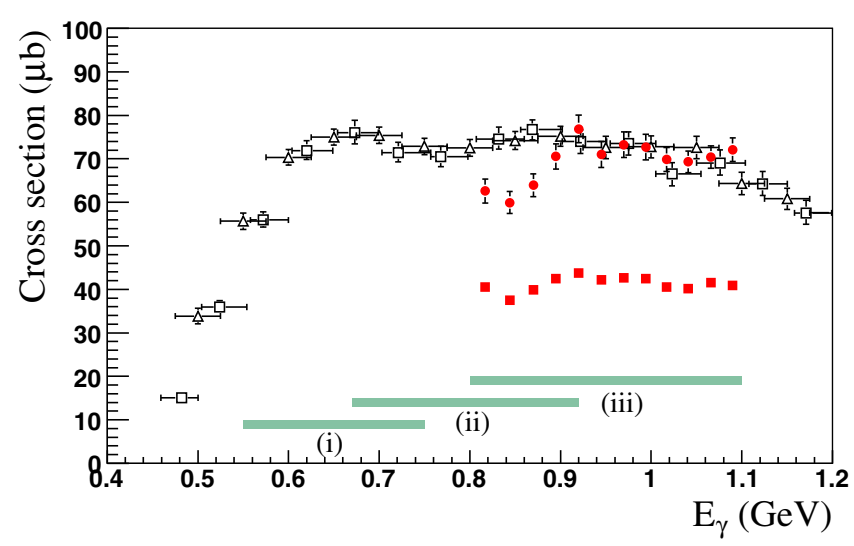

Fig. 1. Cross sections for $\pi^{+} \pi^{-}$photoproduction on the free proton and bound proton. Closed circles and closed squares show the results of this work for the free proton and the bound proton, respectively. Open triangles and open squares show the results for the free proton reported of ABBHHM [13] and SAPHIR [14], respectively. Three bars show the energy regions. The region (i) is the lowest energy region and we proposed the experiment with NKS2 in this region. We took the data in the region (ii) with NKS2; and (iii) with NKS and NKS2.

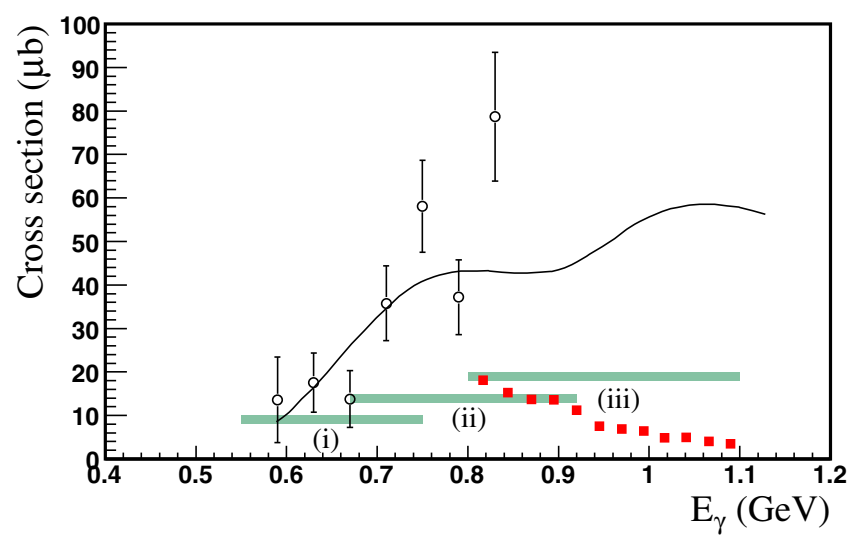

Fig. 2. Cross sections for double $\Delta$ photoproduction on the deuteron. Closed squares show our results. Open circles show the results by Asai et al [2]. Solid curve show the theoretical calculation by Gómez Tejedor et al [3]. The notation of the bars are the same as in Fig. 1

energy region of the tagged photon is from $0.55 \mathrm{GeV}$ to $0.75 \mathrm{GeV}$ and $0.67 \mathrm{GeV}$ to $0.92 \mathrm{GeV}$ for the electron energy as $0.82 \mathrm{GeV}$ and $1.0 \mathrm{GeV}$, respectively. The redundant ranges are set between two neighboring energy regions for checking the consistency of the results. The ABC effect for $p n \rightarrow$ $d \pi^{0} \pi^{0}$ is observed at the energy range of $0.9<T_{p}<1.4 \mathrm{GeV}$ at CELSIUS-WASA[7,8]. It corresponds to the photon energy region in $0.45<E_{\gamma}<0.7 \mathrm{GeV}$ for the same center-of-mass energy. Therefore, the measurement in the lower energy is the matter of importance for the investigation of the $\mathrm{ABC}$ effect in the photon induced reactions. The experiment in the lower energy regions will be performed in the near future.

This work is supported by Grants-in-Aid for Scientific Research from Japan Society for the Promotion of Science (JSPS) and The Ministry of Education, Culture, Sports, Science and Technology(MEXT), Nos. 09304028, 12002001, 14740150, and 16GS0201 and by the Grant Agency of the Czech Republic under Grant 202/05/2142 and 202/08/0984. 
EPJ Web of Conferences

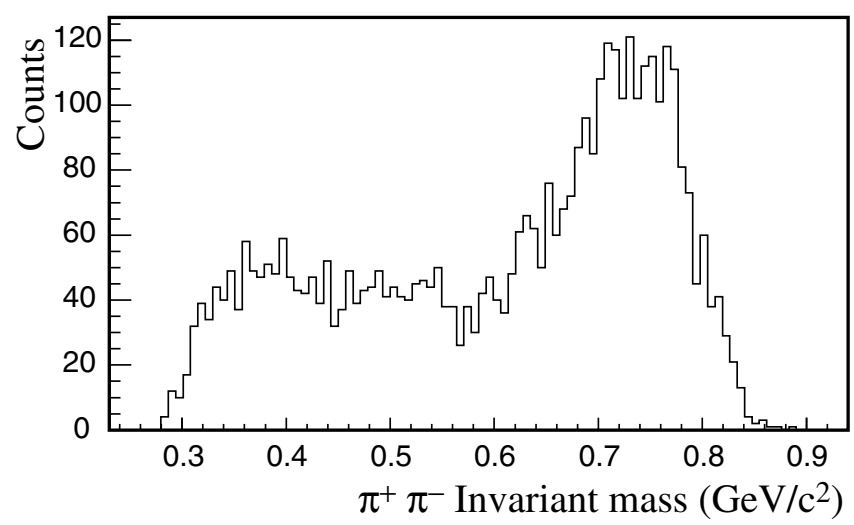

Fig. 3. Invariant mass of the $\pi^{+} \pi^{-}$in the $\pi^{+} \pi^{-} d$ final state.

\section{References}

1. A. Fix and H. Arenhövel, Eur. Phys. J. A 25115.

2. M. Asai, et al., Z. Phys. A 344 (1993) 335.

3. J. A. Gómez Tejedor, E. Oset and H. Toki, Phys. Lett. B 346 (1995) 240.

4. N. E. Booth, A. Abashian, and K. M. Crowe Phys. Rev. Lett. 7 (1961) 35.

5. T. Risser and M. D. Shuster, Phys. Lett. B 43 (1973) 68.

6. C. A. Mosbacher and F. Osterfeld, nucl-th/9903064.

7. M. Bashkanov, et al., Phys. Lett. B 637 (2006) 223.

8. M. Bashkanov et al., Phys. Rev. Lett. 102 (2009) 052301.

9. S. Keleta, et al., Nucl. Phys. A 825 (2009) 71.

10. K. Hirose et al., Phys. Lett. B 674 (2009) 17.

11. H. Yamazaki et al., Nucl. Instrum. and Meth. A 536 (2005) 70.

12. K. Tsukada et al., Phys. Rev. C 78 (2008) 014001.

13. Aachen-Berlin-Bonn-Hamburg-Heidelberg-München Collaboration, Phys. Rev. 175 (1968) 1669.

14. C. Wu et al., Eur. Phys. J. A 23 (2005) 23. 